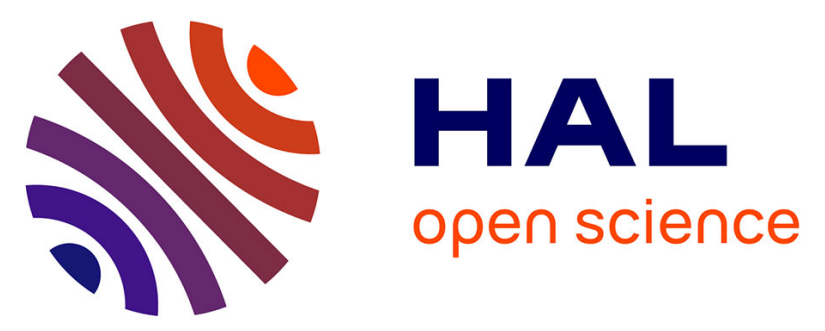

\title{
3D-FDTD-based hybridisation technique for wave interaction with scatterers in layered media
} Julien Vincent, Pierre Borderies, Jean-René Poirier, Vincent Gobin

\section{To cite this version:}

Julien Vincent, Pierre Borderies, Jean-René Poirier, Vincent Gobin. 3D-FDTD-based hybridisation technique for wave interaction with scatterers in layered media. Electronics Letters, 2016, 52 (8), pp.573-574. 10.1049/el.2015.4300 . hal-01407462

\section{HAL Id: hal-01407462 \\ https://hal.science/hal-01407462}

Submitted on 4 Oct 2021

HAL is a multi-disciplinary open access archive for the deposit and dissemination of scientific research documents, whether they are published or not. The documents may come from teaching and research institutions in France or abroad, or from public or private research centers.
L'archive ouverte pluridisciplinaire HAL, est destinée au dépôt et à la diffusion de documents scientifiques de niveau recherche, publiés ou non, émanant des établissements d'enseignement et de recherche français ou étrangers, des laboratoires publics ou privés. 


\section{D-FDTD-based hybridisation technique for wave interaction with scatterers in layered media}

\author{
J. Vincent ${ }^{\bowtie}$, P. Borderies, J.R. Poirier and V. Gobin
}

A simple method for the computation of 3D scatterers remotely located from the source with layered media is presented. This domain decomposition method is based on the total-field/scattered-field formulation of the 3D finite-difference in time-domain method. The electromagnetic field along the propagation path is not directly involved; it focuses only on the electromagnetic field in the surroundings of the scatterer. The incident electromagnetic field radiated from the source is computed separately with an adaptive algorithm and then applied on the Huygens' surface, leading to the proposed hybridisation technique.

Introduction: Computing the electromagnetic field far from the source with the finite-difference in time-domain (FDTD) method can be hard to achieve because of the numerical dispersion in large-scale problems. In $[1,2]$, a very large $2 \mathrm{D}$ computational domain is developed to evaluate the electric field along a propagation path. However, this technique does not take into account the effects of the 3D topography because of the amounts of space and time needed.

The domain decomposition method based on the total-field/scatteredfield formulation (TF/SF) [3] is a solution which can overcome the large-scale problem. This formulation was first used to illuminate scattering bodies in free space with a plane wave [4, 5]. The incident electromagnetic field is known and applied on a 3D closed surface named Huygens' surface. Later the ground is included in [6] and in [7] where an incident plane wave creates transmitted and reflected plane waves by hitting the surface.

In this Letter, the TF/SF formalism is used in order to avoid the propagation of the electromagnetic field radiated by a source and to focus on the interaction with 3D scatterers which can be above, on or under the surface of a layered media. The incident electromagnetic field is known everywhere in space and time or can be computed with another method or software and then applied on the six faces of the Huygens' surface.

TF/SF FDTD formulation with a media: From the linearity of Maxwell's equations, one can decompose the physical total electric field $\boldsymbol{E}_{\text {TOT }}$ and magnetic field $\boldsymbol{H}_{\text {TOT }}$ as

$$
E_{\mathrm{TOT}}=E_{\mathrm{INC}}+E_{\mathrm{SCAT}}, \quad H_{\mathrm{TOT}}=H_{\mathrm{INC}}+H_{\mathrm{SCAT}}
$$

where $\boldsymbol{E}_{\mathbf{I N C}}$ and $\boldsymbol{H}_{\mathbf{I N C}}$ are the incident field components. As stated above, their values are known everywhere in the computation space and at each time step. These are the fields that would exist in all concerned media, without any other object in the computation space. $\boldsymbol{E}_{\text {SCAT }}$ and $\boldsymbol{H}_{\text {SCAT }}$ are the unknown SF components created by the introduction of at least one scatter. Thus, the TF/SF FDTD formalism creates two distinct regions in the computation space bounded by the Huygens' surface (see Fig. 1). Inside the surface, the total electromagnetic field is propagating in the region, and outside the Huygens' surface is the volume where only the SF is taken into account.

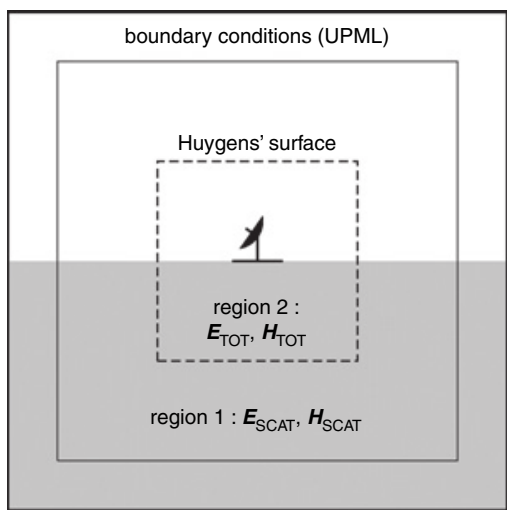

Fig. 1 Representation of TF/SF FDTD formalism. Antenna receiver is located inside volume bounded by Huygens' surface (dashed line) in region 2 (TF). Region 1 (SF) is bounded by boundary conditions (UPML)
As an example, Fig. 2 shows the case of a vertical current element and an antenna receiver at the bottom of a valley, computed with an infinite half-space with the two FDTD formalisms. The receiver is located at several wavelengths from the source. Fig. $2 a$ shows the entire domain which is meshed and computed with the usual 3D FDTD algorithm. The TF/SF formulation is represented in Figs. $2 b$ and $c$. The incident field components are obtained in the case of an infinite flat ground and applied on the Huygens' surface (dashed line) in Figs. $2 b$, and Fig. $2 c$ shows the scattering object considered inside the Huygens' surface.

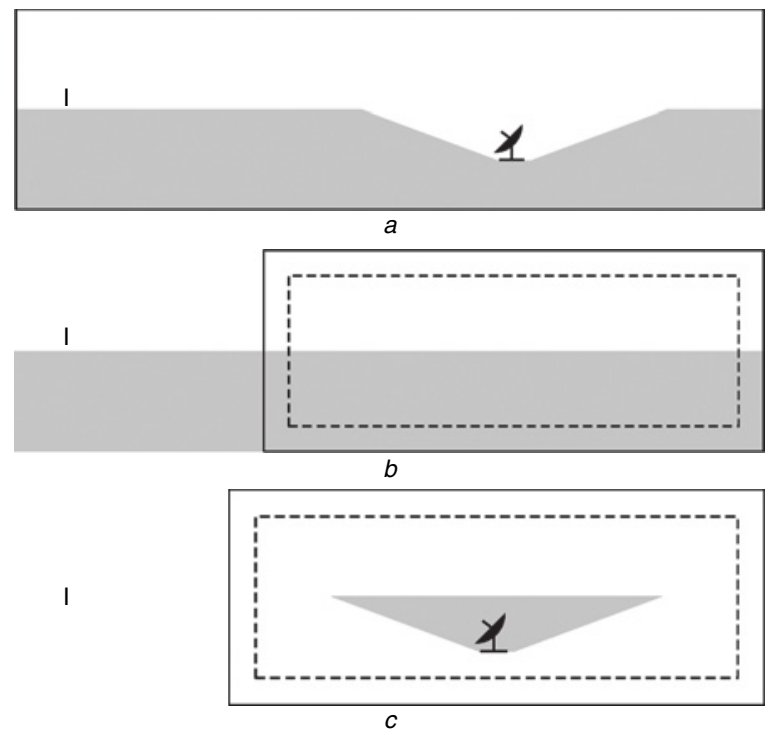

Fig. 2 FDTD computations of radiating current element and antenna receiver at bottom of valley, with regular algorithm and with TF/SF formalism. Scattering object is placed inside the Huygens' surface (dashed line)

$a$ Entire domain computation with usual FDTD algorithm

$b$ Geometry considered in TF/SF formalism, incident electromagnetic field is applied on Huygens' surface

$c$ Scattering object considered in TF/SF formalism with media

As the source is remotely located from the scattering object in the TF/ SF formalism and the propagation path is not computed, the computation time and the memory space needed are obviously reduced. Nevertheless, the values of the incident electromagnetic field applied on all the mesh points of the Huygens' surface at all time steps have to be reasonably accurate.

Incident wave source for TF/SF FDTD formalism with a media: From (1) and from the free-space TF/SF FDTD formulation given in equations (5.48a) to (5.59b) of [3] (pp. 204-210), one can find the assumed known correction terms used in the TF/SF FDTD formulation with a media. Caution should be taken with the coefficients since the dielectric parameters of the media $\left(\varepsilon_{0} \varepsilon_{\mathrm{r}}, \sigma, \mu_{0} \mu_{\mathrm{r}}, \sigma^{*}\right)$ are involved in the computations. In this Letter, only media with relative permeability $\mu_{\mathrm{r}}=1$ and without magnetic loss $\left(\sigma^{*}=0\right)$ are considered.

Obtained from (5.48a) and (5.51a) of [3], (2) and (3) show the coefficients which have to be included on one face of the Huygens' surface, in order to consider a media in the TF/SF formulation. The 22 other formulas which correct the electromagnetic field at each halved time step on the whole Huygens' surface can be easily written from the two following equations and from [3]

$$
\begin{aligned}
& \boldsymbol{E}_{\boldsymbol{x}, \mathbf{T O T}} \mid \begin{array}{c|c}
n+1 \\
i, j_{0}, k
\end{array}=\left\{\begin{array}{l}
\boldsymbol{E}_{\boldsymbol{x}, \mathbf{T O T}} \mid \begin{array}{c}
n+1 \\
i, j_{0}, k
\end{array}
\end{array}\right\} \\
& -\frac{2 \Delta t}{(2 \varepsilon+\sigma \Delta t) \Delta y} \boldsymbol{H}_{z, \mathbf{I N C}} \mid \begin{array}{c}
n+\frac{1}{2} \\
i, j_{0}-1 / 2, k
\end{array} \\
& \boldsymbol{H}_{z, \mathbf{S C A T}} \mid \begin{array}{c|c}
n+1 / 2 \\
i, j_{0}-1 / 2, k
\end{array}=\left\{\begin{array}{ll}
\boldsymbol{H}_{z, \mathbf{S C A T}} & \begin{array}{c}
n+1 / 2 \\
i, j_{0}-1 / 2, k
\end{array}
\end{array}\right\} \\
& -\frac{\Delta t}{\mu_{0} \Delta y} \boldsymbol{E}_{x, \mathbf{I N C}} \mid \begin{array}{c}
n \\
i, j_{0}, k
\end{array}
\end{aligned}
$$

In (2) and (3), $\boldsymbol{E}_{\mathbf{I N C}}$ and $\boldsymbol{H}_{\mathbf{I N C}}$ are the known incident fields applied on 
the whole Huygens' surface at each halved time step. When their values are in the time domain, the pulse is introduced directly in the algorithm. In frequency domain (e.g. the electromagnetic field radiated at a frequency by an antenna, obtained with the boundary integral equation method), one has to use (4) and (5) for a time harmonic notation $\mathrm{e}^{+\mathrm{j} \omega t}$ :

$$
\begin{aligned}
& \boldsymbol{E}^{\mathrm{FDTD}}(x, y, z, t)=\mathfrak{R}\left\{\boldsymbol{E}^{\mathrm{BIE}}(x, y, z) \cdot \mathrm{e}^{+\mathrm{j} \omega t} \cdot\left(1-\mathrm{e}^{-\alpha t}\right)\right\} \\
& \boldsymbol{H}^{\mathrm{FDTD}}(x, y, z, t)=\mathfrak{R}\left\{\boldsymbol{H}^{\mathrm{BIE}}(x, y, z) \cdot \mathrm{e}^{+\mathrm{j} \omega t} \cdot\left(1-\mathrm{e}^{-\alpha t}\right)\right\}
\end{aligned}
$$

where $\boldsymbol{F}^{\text {FDTD }}$ (respectively $\boldsymbol{F}^{\mathrm{BIE}}$ ) is the electromagnetic field applied in FDTD (respectively boundary integral equation) and the term $\left(1-\mathrm{e}^{-\alpha t}\right)$ is used to prevent the rapid changes of values at the beginning of the algorithm. The parameter $\alpha$ is chosen by the user such that the signal has reached its maximum amplitude after a few initialisation periods.

Computation of a receiver antenna far from the source: As an example, a $1125 \mathrm{~m}$ long vertical wire used as a receiver is located at $375 \mathrm{~m}$ over a medium wet flat ground with relative permittivity $\varepsilon_{\mathrm{r}}=15$ and conductivity $\sigma=10^{-3} \mathrm{~S} \mathrm{~m}^{-1}$. The source is remotely located at $23.75 \mathrm{~km}$ from this receiver and $25 \mathrm{~m}$ above the ground. It is a $25 \mathrm{~m}$ long infinitesimal vertical current element $\left(I_{0}=325.76 \times 10^{-6}+j \times 51.581 \mathrm{~A}\right)$ radiating at the frequency $f_{0}=100 \mathrm{kHz}$. The FDTD computation space is a 100 cells side cube with the mesh size $\Delta x=\Delta y=25 \mathrm{~m}$ and $\Delta z=75 \mathrm{~m}$. The ground surface $(z=0 \mathrm{~m})$ is located at the middle of the $z$-directed cells. The incident electromagnetic field in both the air and the ground is computed by a numerical integration of Sommerfeld's integrals with an adaptive algorithm [8]. These values are applied on the Huygens' surface surrounding the receiver. The volume inside the Huygens' surfaces is a 60 cells side cube centred in the 3D FDTD domain. The parameter $\alpha$ is chosen such that $99 \%$ of the signal is reached after five periods (i.e. $\left.\left[1-\exp \left(-\alpha \times 5 / f_{0}\right)\right]=0.99\right)$. Uniaxial-PMLs are used to terminate the $3 \mathrm{D}$ computational domain [9] and the thin wire $(r=5 \mathrm{~mm})$ is computed with the wire formalism of Holland and Simpson [10]. As shown in Fig. 3, the current observed in the middle of the wire receiver needs seven periods to be considered as a sinusoidal waveform. These values are compared with the current obtained by computing the total scene with the boundary equation method of the solver FEKO. The results are in good agreement (relative error of $1.59 \%$ in the sinusoidal waveforms).

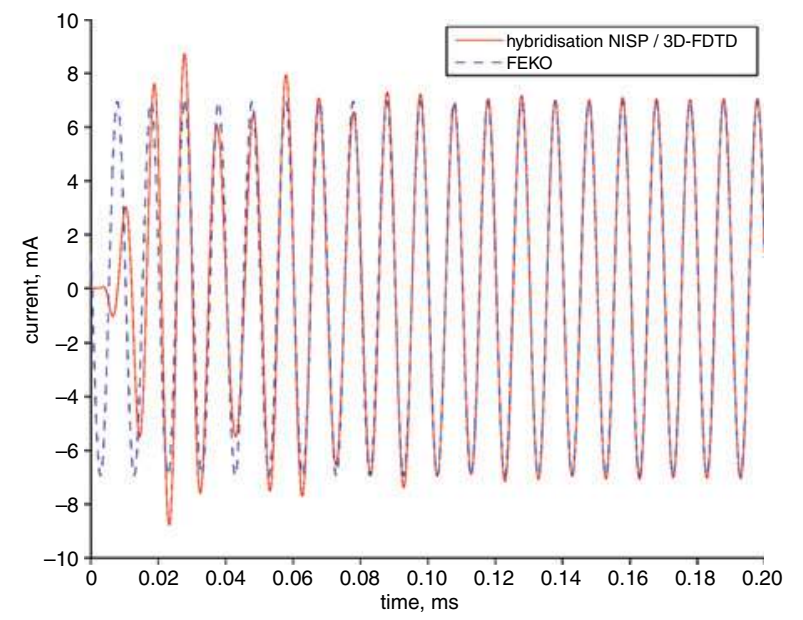

Fig. 3 Current in middle of antenna receiver, located at $23.75 \mathrm{~km}$ from source
Conclusion: A 3D-FDTD-based hybridisation technique is presented for wave interaction with scatterers in layered media. It rests on the $\mathrm{SF}-\mathrm{TF}$ formalism with a media. The incident electromagnetic field is known everywhere in space at each time step and applied on the Huygens' surface. This domain decomposition technique is very useful to avoid large domain computations and to focus on the object located upon or inside the layered media.

Acknowledgments: This work was supported by Direction Générale de l'Armement (DGA) and Office National d'Études et de Recherches Aérospatiales (ONERA).

(C) The Institution of Engineering and Technology 2016 Submitted: 11 December 2015 E-first: 14 March 2016 doi: $10.1049 / \mathrm{el} .2015 .4300$

One or more of the Figures in this Letter are available in colour online. J. Vincent and J.R. Poirier (Université de Toulouse, INPT, UPS, CNRS, LAPLACE, ENSEEIHT, Toulouse Cedex 7, France)

凶E-mail: vincent@laplace.univ-tlse.fr

P. Borderies and V. Gobin (ONERA - The French Aerospace Lab, 31055 Toulouse, France)

\section{References}

1 Zhou, L.L., Xi, X.L., Liu, J.F., and Yu, N.M.: 'LF ground-wave propagation over irregular terrain', IEEE Trans. Antennas Propag., 2011, 59, (4), pp. 1254-1260, doi: 10.1109/TAP.2011.2109693

2 Zhou, L.L., Xi, X.L., and Du, Y.X.: 'Application in low-frequency ground-wave propagation of parallel FDTD based GPU'. 10th Int Symp. on Antennas Propagation EM Theory (ISAPE2012), Xi'an, China, October 2012, pp. 917-920, doi: 10.1109/ISAPE.2012.6408922

3 Taflove, A., and Hagness, S.: 'Computational electrodynamics: the finite-difference time-domain method' (Artech House, Norwood, MA, USA, 2005, 3rd edn.)

4 Merewether, D.E., Fisher, R., and Smith, F.W.: 'On implementing a numeric Huygens' source scheme in a finite difference programme to illuminate scattering bodies', IEEE Trans. Nucl. Sci., 1980, 27, pp. 1829-1833, doi: 10.1109/TNS.1980.4331114

5 Umashankar, K.R., and Taflove, A.: 'A novel methjod to analyze electromagnetic scattering of complex objects', IEEE Trans. Electromagn. Compat., 1982, 24, pp. 397-405, doi: 10.1109/TEMC.1982.304054

6 Huang, Z., Demarest, K., and Plumb, R.: 'Ground-penetrating radar antenna modeling'. Geoscience and Remote Sensing Symp., 1996, IGARSS '96, 'Remote Sensing for a Sustainable Future', May 1996, Vol. 1, pp. 778-780, doi: 10.1109/IGARSS.1996.516472

7 Demarest, K., Plumb, R., and Huang, Z.: 'FDTD modeling of scatterers in stratified media', IEEE Trans. Antennas Propag., 1995, 43, (10), pp. 1164-1168, doi: 10.1109/8.467657

8 Vincent, J., Borderies, P., Gobin, V., and Poirier, J.-R.: 'Modelling the effects of realistic environments in the nearfield and farfield of low frequency antennas with 3D FDTD method'. 2014 Int. Conf. on Numerical Electromagnetic Modeling and Optimization for RF, Microwave, and Terahertz Applications (NEMO), Pavia, Italy, May 2014, pp. 1-4, doi: 10.1109/NEMO.2014.6995711

9 Gedney, S.D.: 'An anisotropic perfectly matched layer-absorbing medium for the truncation of FDTD lattices', IEEE Trans. Antennas Propag., 1996, 44, (12), pp. 1630-1639, doi: 10.1109/8.546249

10 Holland, R., and Simpson, L.: 'Finite-difference analysis of EMP coupling to thin struts and wires', IEEE Trans. Electromagn. Compat., 1981 EMC-23, (2), pp. 88-97, doi: 10.1109/TEMC.1981.303899 